\title{
Factors Associated With Failure of Weaning From Long- term Mechanical Ventilation After Cardiac Surgery
}

\author{
Emilia NozAwA, ${ }^{1}$ PT, Estela AzEKA, ${ }^{1}$ MD, Maria Ignêz Z \\ Zanetti FELTRIM, ${ }^{1}$ PT, and José Otávio Costa AULER Júnior, ${ }^{1}$ MD
}

\begin{abstract}
SUMMARY
The purpose of this prospective, quantitative, comparative study, conducted at the 55 bed cardiothoracic intensive care unit of the Heart Institute (InCor), University of Sao Paulo Medical School, was to identify factors involved in the weaning of patients who require long-term ( $>10$ days) mechanical ventilation after cardiac surgery.

The subjects included all patients who underwent open-heart surgery with cardiopulmonary bypass during a 10 month period from April 2000 to January $2001(n=946)$. From this group, $52(5.7 \%)$ patients who required a tracheotomy for the management of long-term mechanical ventilation after cardiac surgery with cardiopulmonary bypass were selected. Pre-, intra- and postoperative data from patients who were not successfully weaned after reintubation and who underwent an elective tracheotomy were compared.

Parameters of respiratory mechanics such as respiratory complications, oxygenation, and cardiac, renal, and neurological function were evaluated. Weaning success was defined as the ability of a patient to tolerate 48 hours without pressure or flow support from a mechanical ventilator. A patient was considered to have failed weaning if they died or remained under ventilation for more than 8 weeks. Of the 52 patients studied, 25 were successfully weaned, 21 died, and 6 remained ventilated for more than 8 weeks. We found significant statistical differences $(P<0.05)$ between the groups with respect to success or failure in $\operatorname{LVEF}(P=0.0035)$, the need for vasoactive agents $(P=0.0018)$, and renal failure $(P=0.002)$. Parameters of respiratory mechanics and oxygenation (eg, static airway compliance, airway resistance) did not influence the success or failure of weaning. There was a significant difference in relation to the presence of pneumonia $(P=0.0086)$ between the two groups. Although neurological complications were more frequent in patients in the weaning success group, the failure group had lower GCS scores, which is indicative of worse prognoses.

It is concluded that cardiac dysfunction, the need for dialysis, and pneumonia are determinants for weaning failure in patients undergoing long-term mechanical ventilation after cardiac surgery. (Int Heart J 2005; 46: 819-831)
\end{abstract}

Key words: Long-term, Mechanical ventilation, Weaning, Cardiac surgery

From the ${ }^{1}$ Division of Anesthesia, Surgery and Physiotherapy, Heart Institute (InCor) Hospital das Clinicas, University of Sao Paulo School of Medicine, Brazil.

Address for correspondence: Emilia Nozawa, PT, the Division of Anesthesia, Surgery and Physiotherapy, Heart Institute (InCor) Hospital das Clinicas, University of São Paulo School of Medicine, Av. Dr. Enéas de Carvalho Aguiar 44, $2^{\circ}$ andar; CEP: 05403-000, São Paulo, Brazil.

Received for publication January 11, 2005

Revised and accepted May 13, 2005. 
Postoperative cardiac patients are generally able to resume spontaneous ventilation as soon as they have recovered from the anesthesia; therefore the mode of ventilation should have little impact on the decision to extubate these patients. ${ }^{1)}$ During the time under ventilatory support, cardiovascular and other systems are thought to recover from the profound physiologic disturbances induced by cardiopulmonary bypass. However, recent efforts to improve the costeffectiveness of surgical procedures, combined with revised anesthetic techniques for postoperative sedation and analgesia, ${ }^{2}$ have resulted in a trend toward early weaning from ventilatory support after cardiac surgery.

This strategy has been proven safe in select patients and is now recommended in the care of uncomplicated patients after cardiac surgery, shortening the length of stay in the intensive care unit (ICU) without adversely affecting morbidity or mortality rates.-5) However, approximately $3 \%$ to $6 \%$ of the patients admitted to the adult ICU require prolonged mechanical ventilation, and criteria and strategies for their successful weaning are less clear. ${ }^{6}$ Parameters of respiratory mechanics and oxygenation are commonly used to wean patients with chronic obstructive pulmonary disease (COPD) and other pulmonary disorders from long-term mechanical ventilation. ${ }^{7-9)}$ Yet, as we have previously shown, ${ }^{10)}$ arterial blood gas analysis and the determination of vital capacity and minute volume often fail to predict extubation success in patients after cardiac surgery. Others have identified advanced age, female sex, (extended) time of extracorporeal circulation, cardiac dysfunction, and low cardiac output as factors associated with the failed weaning of cardiac patients from long-term ventilation ${ }^{2,45}$ and postoperative cardiac dysfunction and neurological alterations, as factors contributing to their high mortality. ${ }^{6)}$ But few reports have described parameters of respiratory mechanics, oxygenation, and organ function associated with successful weaning in this population. Therefore, the primary objective of the present study was to analyze pre-, intra- and postoperative data from 52 patients who underwent tracheostomy and long-term mechanical ventilation after cardiac surgery to identify factors associated with successful or failed weaning.

\section{METHODS}

Inclusion and exclusion criteria: All enrolled patients had undergone cardiac surgery with extracorporeal circulation and were tracheostomized and ventilated for an average of 10 days (between April and December 2001) in the Surgical Intensive Care Unit at the Heart Institute (InCor) of the University of Sao Paulo Medical School. Patients who had been diagnosed with sepsis or mediastinitis were excluded from the study. The Scientific Committee of InCor and the Committee 
of Ethics in Research at our institution approved the study, and informed consent was obtained from each patient.

Ventilator parameters: After tracheotomy, the patients were sedated with intravenous midazolam at a dose of $0.1 \mathrm{mg} / \mathrm{kg}$; muscular relaxation was induced with pancuronium bromide at a dose of $0.15-0.2 \mathrm{mg} / \mathrm{kg}$ for the measurement of respiratory system mechanics. A Veolar-Hamilton ventilator (Switzerland) was used and adjusted to the parameters. The ventilator parameters were modified only to normalize the oxygenation and acid-base status. Tidal volumes were chosen based on lean body weight, using 6 to $8 \mathrm{~mL} / \mathrm{kg}$. The peak inspiratory pressure, volume minute, and respiratory rate (RR) were obtained from the ventilator indicators. The plateau pressure was obtained by switching the patient to a squarewave inspiratory pattern combined with a 0.5 second inspiratory pause. The inspired fraction of oxygen $\left(\mathrm{FiO}_{2}\right)$ and the end-expiratory pressure were adjusted to maintain a peripheral oxygen saturation $>95 \%$ until achievement of a lower limit of oxygen concentration of 0.4 and a positive end expiratory pressure (PEEP) of $5 \mathrm{cmH}_{2} \mathrm{O}$. After adjusting the mechanical ventilator, the following measurements were obtained directly from the panel: tidal volume, peak inspiratory pressure, minute volume, RR, plateau inspiratory pressure, PEEP, and inspiratory flow.

Static compliance was calculated by dividing the tidal volume delivered during the plateau maneuver by the plateau pressure minus any PEEP that the patient was receiving. Airway resistance was calculated by dividing the difference between the peak and plateau pressures by the inspiratory flow, again using the square wave inspiratory flow pattern. Three measurements for the compliance and resistance were made, and the average value was recorded for each. The dead space to tidal volume was measured from arterial blood gas data using a noninvasive monitor capnograph/pulse oximeter which verifies oxygenation $\left(\mathrm{CO}_{2} \mathrm{SMO}\right.$ Plus-Novametrix Medical Systems, Wallingford, CT, USA).

Weaning protocol: A weaning trial entailed changing the ventilation mode from SIMV to the continuous positive airway pressure (PEEP) of $5 \mathrm{cmH}_{2} \mathrm{O} R R=2$ breaths/min, $\mathrm{PSV}=10 \mathrm{cmH}_{2} \mathrm{O}$, and $\mathrm{FiO}_{2}=0.4$.

The following parameters were used to assess the suitability for weaning from the ventilator: hemodynamic stability; $\mathrm{RR}<25$ breaths/min, peripheral arterial oxygen content/fraction of inspired oxygen $\left(\mathrm{PaO}_{2} / \mathrm{FiO}_{2}\right)>200$; carbon dioxide arterial pressure between 35 and $45 \mathrm{mmHg}$; maximum inspiratory pressure > $-25 \mathrm{cmH}_{2} \mathrm{O}$, tidal volume $>5 \mathrm{~mL} / \mathrm{kg}$; and maintained arterial oxygen saturation $\left(\mathrm{SaO}_{2}\right) \geq 95 \%$. Patients were considered to be successfully weaned if they could tolerate 48 consecutive hours without any pressure or flow support from a mechanical ventilator. Patients who failed weaning included those who remained 
under mechanical ventilation for $>8$ weeks or who died within 42 days of the weaning trial.

Data and measurements: Preoperative data evaluated consisted of demographic and prior health information, including any history of diabetes mellitus, hypertension, smoking, arterial vascular disease, chronic obstructive pulmonary disease, asthma, myocardial infarction, cerebral vascular accidents, renal insufficiency, or previous cardiac surgery. The Parsonnet score was used to quantify preoperative risk. ${ }^{11)}$ Intraoperative variables assessed included the type of surgery, the duration of the cardiopulmonary bypass, and the frequency of cardiac complications (e.g., low cardiac output requiring intraaortic balloon pumping, arrhythmias, reexploration for persistent bleeding). The physiologic data for the Acute Physiology and Chronic Health Evaluation (APACHE) II score ${ }^{12)}$ were collected during the 24 hours just before the tracheotomy day. Postoperative variables assessed included parameters of respiratory mechanics and oxygenation as well as the frequency of cardiac dysfunction, pulmonary complications (e.g., pleural effusion, atelectasis, pneumonia), renal failure requiring dialysis, diaphragmatic paralysis, neurological alterations, and low Glasgow Coma Scale (GCS) scores.

Definitions of organ dysfunction: Cardiac dysfunction was defined as: a) a low cardiac output syndrome (cardiac index $<1.8 \mathrm{~L} / \mathrm{min} / \mathrm{m}^{2}$ ) in patients receiving vasoactive agents, requiring intra-aortic balloon pump support, and/or presenting with an ejection fraction of $<50 \%$, diagnosed by Doppler echocardiography.

Neurological dysfunction was identified by clinical examination and confirmed by visualization of a focal brain lesion on a computerized tomography scan. Renal dysfunction was defined as: a) dialysis access line or ultrafiltration requirement.

Hypoxemia was defined as a $\mathrm{PaO}_{2} / \mathrm{FiO}_{2} \leq 150$ torr. Chronic obstructive pulmonary disease (COPD) or asthma was diagnosed based on history and the need for bronchodilator therapy. Diaphragmatic dysfunction was considered when the chest radiographs showed elevation of diaphragmatic cupules, and diaphragmatic paralysis was confirmed by ultrasound exam. Pulmonary complications were considered in patients whose computerized tomography scans showed isolated atelectasis, compression atelectasis, pleural effusion, and pneumonia. Pleural effusion was defined as small if the pleural fluid obliterated the costophrenic angle or obscured the lower lung zone, moderate if the fluid opacified the lower and middle lung zones and was visualized on a maximum of five slices, and large if $2 / 3$ of the hemithorax was opacified and the effusion was visualized on more than five slices. Bacterial pneumonia was defined as the presence of a new onset of purulent tracheobronchial secretion, rales on physical examination, a chest radiograph showing new or progressive pulmonary infiltrates, and an air bronchogram. These findings were confirmed by computerized tomography scan. 
Statistical analysis: All variables are presented as the mean \pm standard deviation. When the variables were approximately normally distributed, the Student $t$ test or Wilcoxon rank sum test was used to analyze the means related to weaning failure. The relationships between the groups who experienced weaning success or failure groups were analyzed by the chi-square test, likelihood ratio, or the Fisher exact test. For univariate analyses that were statistically significant, the stepwise multiple logistic regression model was adjusted. Standard formulas were used to calculate the sensitivity and specificity. Probability values $<0.05$ were considered to be statistically significant.

\section{RESULTS}

Of 946 patients who had undergone heart surgery with cardiopulmonary bypass over a 10-month period, $52(2 \%)$ were prospectively evaluated. Of these 52 patients, 25 were successfully weaned from mechanical ventilation and 27 failed attempts at weaning (a total of 21 died and 6 remained on the ventilator for more than 8 weeks). The surgical procedures performed on the patients included $18(34.6 \%)$ coronary artery bypass grafts, $7(13.4 \%)$ redo coronary artery bypass grafts, $9(17.3 \%)$ valve replacements, $8(15.3 \%)$ redo valve replacements, 6 $(11.5 \%)$ CAGB or valve replacement associated with another procedure, 2 $(3.8 \%)$ thoracic aorta surgeries, and $2(3.8 \%)$ corrections of an interventricular septal defect after acute myocardial infarction. We found no statistically difference in type of surgical procedure between the two groups.

Table I. Patient Demographic and Clinical Characteristics

\begin{tabular}{lccc}
\hline Characteristic & Failure $^{\mathrm{a}}(n=27)$ & Success $^{\mathrm{a}}(n=25)$ & $P$ Value $^{\mathrm{b}}$ \\
\hline Mean age (years) & $62.2 \pm 13.2$ & $64.0 \pm 12.6$ & $\mathrm{NS}$ \\
Sex (male/female) & $12 / 25$ & $12 / 27$ & $\mathrm{NS}$ \\
Tracheotomy prior to enrollment in the study (days) & $12 \pm 7$ & $10 \pm 4$ & $\mathrm{NS}$ \\
Mean duration of surgery (hours) & $7 \pm 1.5$ & $7 \pm 1.5$ & $\mathrm{NS}$ \\
Parsonnet score & $15 \pm 6$ & $12 \pm 7$ & $\mathrm{NS}$ \\
Mean cardiopulmonary bypass time (minutes) & $113.7 \pm 49.2$ & $122.5 \pm 49.2$ & $\mathrm{NS}$ \\
APACHE II score & $19.4 \pm 7.3$ & $19.4 \pm 7.3$ & $\mathrm{NS}$ \\
Duration of mechanical ventilation (days) & $33 \pm 17$ & $30 \pm 8$ & $\mathrm{NS}$ \\
Mean duration of stay in ICU (days) & $40 \pm 12$ & $38 \pm 10$ & $\mathrm{NS}$ \\
\hline
\end{tabular}

ICU indicates intensive care unit; NS, not significant.

APACHEII: Acute Physiology and Chronic Health Evaluation was based on the worst physiologic values during the 24 hours just before the tracheotomy day.

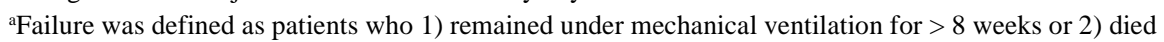
in close proximity to weaning attempts. Success was defined as patients who achieved complete autonomy from ventilator for more than 48 hours.

${ }^{\mathrm{b}} P$ values were determined using the two-tailed paired Student $t$-test. 
Table II. Preoperative and Intraoperative Variables

\begin{tabular}{lccc}
\hline Variable & Failure $^{\mathrm{a}}(n=27)$ & Success $^{\mathrm{a}}(n=25)$ & $P$ Value \\
\hline Preoperative: & 13 & 7 & $\mathrm{NS}$ \\
$\quad$ Systemic arterial hypertension & 13 & 6 & $\mathrm{NS}$ \\
$\quad$ Diabetes mellitus & 2 & 1 & $\mathrm{NS}$ \\
$\quad$ Renal disease & 6 & 5 & $\mathrm{NS}$ \\
$\quad$ Myocardial infarction & 2 & 1 & $\mathrm{NS}$ \\
$\quad$ Chronic obstructive pulmonary disease & 16 & 12 & $\mathrm{NS}$ \\
$\quad$ Smoking & 3 & 1 & $\mathrm{NS}$ \\
$\quad$ Central nerve disorder & 12 & $\mathrm{NS}$ \\
$\quad$ Previous cardiac surgery & 20 & 9 & 0.041 \\
Intraoperative: & & \\
$\quad$ Cardiac complications (low cardiac output, \\
arrhythmias, cardiac arrest and bleeding)
\end{tabular}

Table III. Comparison of Parameters of Respiratory Mechanics and Oxygenation

\begin{tabular}{lccc}
\hline Variable & Failure $^{\mathrm{a}}$ & Success $^{\mathrm{a}}$ & $P$ Value $^{\mathrm{b}}$ \\
\hline Static compliance $\left(\mathrm{mL} / \mathrm{cmHO}_{2}\right)$ & $34 \pm 12.8$ & $37 \pm 13.6$ & $\mathrm{NS}$ \\
Airway resistance $\left(\mathrm{cmHO}_{2} 1 / \mathrm{s}\right)$ & $13.4 \pm 8.1$ & $17.8 \pm 15.6$ & $\mathrm{NS}$ \\
$\mathrm{PaCO}_{2}$ torr & $46 \pm 9.2$ & $44 \pm 7.0$ & $\mathrm{NS}$ \\
$\mathrm{PaO}_{2} / \mathrm{FiO}_{2}$ ratio & $214 \pm 85.4$ & $211 \pm 88$ & $\mathrm{NS}$ \\
$\mathrm{V}_{\mathrm{D}} / \mathrm{V}_{\mathrm{T}}$ ratio & $0.51 \pm 0.11$ & $0.45 \pm 0.15$ & $\mathrm{NS}$ \\
\hline
\end{tabular}

NS indicates not significant; $\mathrm{PaO}_{2}$, peripheral arterial oxygen content; $\mathrm{PaCO}_{2}$, carbon dioxide arterial pressure; $\mathrm{FiO}_{2}$, inspired fraction of oxygen; and $\mathrm{V}_{\mathrm{D}} / \mathrm{V}_{\mathrm{T}}$ ratio, dead space-to tidal volume ratio.

${ }^{a}$ Failure was defined as patients who 1) remained ventilated for $>8$ weeks or 2) died in close proximity to weaning attempts. Success was defined as patients who achieved complete autonomy from the ventilator for more than 48 hours.

${ }^{\text {b}}$ The two-tailed paired Student t-test was used to determine $P$ values.

Table I summarizes the demographic and clinical characteristics, and Table II summarizes the preoperative and intraoperative variables. Comparison of the parameters of respiratory mechanics and oxygenation (static pulmonary compliance, airway resistance, dead space/tidal volume ratio, $\mathrm{PaO}_{2} / \mathrm{FiO}_{2}$ ratio) revealed no statistically significant differences between the group who experienced weaning success and the group who failed to be weaned (Table III). However, a comparison of variables reflecting organ function revealed significant differences in 
Table IV. Comparison of Parameters of Cardiac, Renal, Pulmonary, and Neurological Function

\begin{tabular}{lccc}
\hline Variable & Failure $^{\mathrm{a}}(n=27)$ & Success $^{\mathrm{a}}(n=25)$ & $P$ Value $^{\mathrm{b}}$ \\
\hline LVEF (\%) & $48 \pm 15.1$ & $60 \pm 13.6$ & 0.0030 \\
Use of vasoactive agents & 23 & 11 & 0.0018 \\
Need for dialysis & 18 & 4 & 0.0003 \\
Atelectasis & 23 & 19 & $\mathrm{NS}$ \\
Left pleural effusion & 16 & 14 & $\mathrm{NS}$ \\
Right pleural effusion & 18 & 17 & $\mathrm{NS}$ \\
Pneumonia & 15 & 5 & 0.0086 \\
Diaphragmatic paralysis & 3 & 2 & $\mathrm{NS}$ \\
Neurological changes & 5 & 11 & 0.0467 \\
GCS & $5 \pm 3$ & $11 \pm 4$ & 0.0487 \\
\hline
\end{tabular}

NS indicates not significant; LVEF, left ventricular ejection fraction; and GCS, Glasgow Coma Scale.

${ }^{\text {a}}$ Failure was defined as patients who 1) remained ventilated for $>8$ weeks or 2) died in close proximity to weaning attempts. Success was defined as patients who achieved complete autonomy from the ventilator for more than 48 hours.

${ }^{\mathrm{b}}$ The chi-square test or Fisher exact test was used to determine $P$ values.

Table V. Relationship Between Pneumonia and Other Variables

\begin{tabular}{lcccc}
\hline Variable & Pneumonia & Failure & Success & $P$ Value \\
\hline LVEF $<50$ & 0 & 4 & 4 & 0.018 \\
\multirow{2}{*}{ Need for dialysis } & 1 & 11 & 0 & \\
\multirow{2}{*}{ Left pleural effusion } & 0 & 8 & 4 & NS \\
\multirow{2}{*}{ Right pleural effusion } & 1 & 10 & 0 & \\
\multirow{2}{*}{ Diaphragmatic paralysis } & 0 & 6 & 10 & NS \\
\multirow{2}{*}{ Low Glasgow Coma Score $<9$} & 1 & 10 & 4 & \\
& 0 & 7 & 14 & 0.009 \\
& 1 & 11 & 3 & \\
& 0 & 2 & 1 & NS \\
& 1 & 1 & 1 & \\
& 0 & 2 & 3 & NS \\
\hline
\end{tabular}

NS indicates not significant; LVEF, left ventricular ejection fraction, 0 , pneumonia absent; and 1, presence of pneumonia.

the frequency of cardiac dysfunction, renal failure (i.e., need for dialysis), pneumonia, and neurological alterations between the two groups (Table IV). The presence of pneumonia was associated with pulmonary complications, cardiac dysfunction and dialysis (Table V). A difference in the duration of mechanical ventilation in patients with pneumonia was not observed although there were more patients with pneumonia in the failure group ${ }^{15)}$ than in the success group. ${ }^{5)}$ The multivariate correlation by stepwise logistic regression revealed that pneu- 
Table VI. Multiple Regression

\begin{tabular}{lcclcc}
\hline Variable & Coefficient & Standard error & Odds ratio $(95 \% \mathrm{CI})$ & $\chi^{2}$ & $P$ value \\
\hline Dialysis & 2.89 & 0.93 & $17.24(2.10-107.3)$ & 10.0 & $<0.001$ \\
Pneumonia & 2.50 & 0.95 & $12.29(1.8-79.8)$ & 6.9 & $<0.001$ \\
LEVF & 2.35 & 0.90 & $10.56(1.7-62.4)$ & 6.7 & $<0.001$ \\
\hline
\end{tabular}

LVEF indicates left ventricular ejection fraction; CI, confidence interval; and $\chi^{2}$, chi-square

monia, LEVF and dialysis were factors related to the failure to wean (Table VI).

\section{DISCUSSION}

Pre- and intraoperative clinical factors: In this study, the mean age of the patients (63 years) was not a key factor in determining the success or failure of weaning from long-term mechanical ventilation. Similar results were found by Kolleff, et $a l^{13)}$ who studied patients requiring long-term mechanical ventilation for more than 48 hours after cardiac surgery. These results differ from those in a previous study that showed that age is a powerful predictor of delayed extubation after cardiac surgery. ${ }^{14)}$ In that study, elderly patients were shown to be at a higher risk of cardiovascular disease due to a reduction in cardiac reserve and autonomic tonus and were more likely to experience arrhythmias or hemodynamic instability while recovering from cardiac surgery. ${ }^{14)}$

We also verified that gender does not influence the success or failure of weaning from long-term mechanical ventilation after cardiac surgery. However, female gender is considered to be a risk factor after cardiac surgery because of reduced corporeal surface area, smaller artery diameters, and a reduction in estrogen.

Preoperative variables such as the frequency of systemic arterial hypertension, diabetes mellitus, renal failure, myocardial infarction, chronic obstructive pulmonary disease, smoking, previous cardiac surgery, and central nerve disorders did not significantly differ between the groups who experienced weaning success or failure. Similar results have been reported by Wahl, et al. ${ }^{15)}$ However, our study did reveal a higher frequency of cardiac complications (low cardiac output, arrhythmias, cardiac arrest and bleeding) during the intraoperative period in the group that failed weaning. These results are consistent with the previous identification of intraoperative events such as myocardial failure ${ }^{6}$ and surgical reexploration due to bleeding ${ }^{2)}$ as factors that lead to failure of weaning from mechanical ventilation after cardiac surgery. In our study, the type of surgical pro- 
cedure, the mean duration of the surgery, and the mean CPB time did not appear to affect the success or failure of weaning from long-term mechanical ventilation. Postoperative factors: Our data also demonstrated that 21 patients died before they could be successfully weaned, while the remaining 6 of 27 patients who failed weaning remained on the ventilator for more than 8 weeks. These data are generally consistent with data from previous studies, ${ }^{5,16)}$ except that Engoren, et $a l^{16)}$ reported that a higher percentage of their patients $(83.9 \%)$ were successfully weaned from long-term mechanical ventilation after cardiac surgery.

Cardiac dysfunction has been identified as a factor that makes weaning from mechanical ventilation difficult in patients following cardiac surgery, ${ }^{2,5)}$ as well as constituting a major cause of death. ${ }^{17)}$ Weintraub, et al ${ }^{18)}$ reported that coronary artery bypass patients who experience higher mortality rates are those with evidence of cardiac dysfunction (LVEF < 50\%). Similar results were found by O' Connor, et $a l{ }^{19)}$ who showed that in-hospital mortality was greater in bypass patients with a LVEF $<40 \%$ than in patients with a LVEF $>60 \%$. In our study, a low LVEF was identified as a factor that contributed to the failure of weaning from mechanical ventilation. We verified both a smaller ejection fraction and a greater requirement for vasoactive agents in the failure group compared with the success group.

In patients with respiratory failure, parameters of static pulmonary compliance are recommended for predicting the success of weaning from mechanical ventilation. ${ }^{9)}$ Others factors that may cause low static pulmonary compliance are a sternotomy, insertion of a pleural drain, trauma to the chest wall during dissection, and a decrease in the intercostal blood supply after removal of the internal thoracic artery. ${ }^{20,21)}$ A finding of low pulmonary compliance after cardiopulmonary bypass is well established, likely due to the tendency of interstitial edema, hemorrhage and vascular congestion to increase pulmonary stiffness. ${ }^{22)}$ Our study showed that although low static lung compliance was present, it was not sensitive enough to predict the weaning outcomes of our patients because they usually had normal pulmonary function before the surgery.

The dead space-to-tidal volume $\left(\mathrm{V}_{\mathrm{D}} / \mathrm{V}_{\mathrm{T}}\right)$ ratio may be helpful in weaning patients from long-term mechanical ventilation, especially in conjunction with respiratory mechanics and oxygenation data. However, this ratio was not useful in predicting the success or failure of weaning from long-term mechanical ventilation in our study, because both groups had similar values. Nozawa, et al ${ }^{10)}$ reported similar results in another study involving patients who underwent longterm mechanical ventilation after cardiac surgery.

The $\mathrm{PaO}_{2} / \mathrm{FiO}_{2}$ ratio is known as the oxygenation index and is routinely used as a weaning parameter in early extubation after cardiac surgery at our institution. It is possible to use the $\mathrm{PaO}_{2} / \mathrm{FiO}_{2}$ ratio as an independent parameter, apart from 
the patient's clinical condition, ${ }^{23)}$ without affecting survival during the immediate postoperative period. However, the $\mathrm{PaO}_{2} / \mathrm{FiO}_{2}$ ratio was not identified as a factor predictive of the outcome of weaning patients from long-term mechanical ventilation in the present study.

Pneumonia following cardiac surgery is more frequent in patients with dialysis, reintubation, tracheostomy, and an intra-aortic balloon pump. ${ }^{24)}$ Kollef, et $a l^{24)}$ reported that among patients developing a nosocomial infection, the mortality rates were significantly greater when associated with urinary and wound infections. Bouza, et $a l^{25)}$ have demonstrated that in patients with more than 7 days of intubations, the incidence of pneumonia was $44.1 \%$ after cardiac surgery. Our study demonstrates that pneumonia was frequent $(38.4 \%)$ in patients with mechanical ventilation lasting more than 10 days, and we observed that pneumonia was significantly more frequent in the failure group, probably due to the association of pleural effusion and cardiac dysfunction.

Landymore \& Howell ${ }^{26}$ ) evaluated respiratory complications in patients after myocardial revascularization with internal mammary artery grafts by examining chest radiographs obtained during their first 3 months of convalescence. They found that a high percentage $(92 \%)$ of the patients who had undergone pleural opening and dissection developed a pleural effusion, with reductions in lung volume (atelectasis) and elevation of the left hemidiaphragm. Pleurotomy is more frequent on the left, where either a greater increase in the production of pleural fluid or decrease in lymphatic depuration may lead to left pleural effusions. ${ }^{26}$ ) Consistent with the results of previous studies, the present study revealed a high incidence of pleural effusions in both of our groups who were recovering from cardiac surgery.

The etiology of atelectasis after cardiac surgery with cardiopulmonary bypass is multifactorial. The many possible causes include intravascular microaggregates or blood cell damage-induced leukocyte activation, interstitial pulmonary edema which can be aggravated by volume overload, tissue hypoxia, and altered surfactant activity. ${ }^{27)}$ Singh, et $a l^{28)}$ reported that atelectasis can be explained by sternotomy, bilateral pleurotomy, pleural effusion, and, sometimes, pulmonary trauma during surgery or insertion of a drain in the pleural cavity. Tenling, et $a l^{29)}$ studied 18 patients who underwent coronary artery bypass grafting or valve replacement and verified, by computed tomography, atelectasis in 17 patients $(94.4 \%)$ on the first postoperative day. Similarly, a high incidence of atelectasis (85\%) was found in our study, although $63.8 \%$ of the cases were caused by compression, possibly due to a pleural effusion.

Phrenic nerve lesions are relatively frequent in patients who have undergone cardiac surgery. The main cause of diaphragmatic paralysis is topical cardiac hypothermia, which is used for myocardial protection in patients undergoing car- 
diac surgery. In addition to cold injury to the phrenic nerve, trauma caused during dissection of the internal thoracic artery (needed for adequate positioning and visualization of the coronary artery) may lead to diaphragmatic dysfunction. ${ }^{23)}$ In our study, 3 of the 5 patients who experienced diaphragmatic paralysis were in the weaning failure group, and all of them demonstrated phrenic paralysis.

Neurological complications, when present postoperatively, are one of the most serious complications that contribute to long-term mechanical ventilation after cardiac surgery. The clinical outcome of these patients depends on the degree of the neurological lesions. The mechanisms involved in the genesis of these lesions are multifactorial and include factors such as advanced age, hemodynamic instability, obesity, inflammatory factors/platelet aggregation, cerebral hypothermia, and inflammatory and neurohumoral factors associated with the surgery. ${ }^{30,31)}$ In a recent prospective multicentre study conducted by Roach et al., neurological alterations were documented in 129 of 2108 (6.1\%) patients after coronary artery bypass surgery with extracorporeal circulation. ${ }^{30}$ In the present study, although neurological alterations were more frequent in patients in the weaning success group, patients in the weaning failure group had lower GCS scores and worse prognoses. These findings are consistent with previous reports that neurological complications experienced during coronary bypass procedures lead to poor outcomes. ${ }^{15,32)}$

Acute renal failure is one of the most serious complications in patients after cardiac surgery with extracorporeal circulation. However, only a small percentage $\left(1 \%\right.$ or $2 \%$ ) of the patients who develop acute renal failure require dialysis. ${ }^{33)}$ Despite technological improvements in the procedure and advances in the ICU, the mortality rate in patients with acute renal failure has been high over the last 3 decades, reaching $67 \%$ among patients who required dialysis after cardiac surgery. ${ }^{34)}$ In the present study, we observed a statistically significant difference in the frequency of dialysis between the two groups. We also identified the need for dialysis as a strong predictor (sensitivity of $84 \%$ and specificity of $90 \%$ ) of patients who will fail attempts at weaning from long-term mechanical ventilation after cardiac surgery.

This study also has some limitations. For example, it was conducted at a large referral center performing many operations per year, so the results may not be completely generalizable to a more typical center with a lower volume of cardiopulmonary bypass patients and different practice patterns. In addition, subsequent investigations are needed to confirm the independent association of these factors with multiorgan dysfunction and to demonstrate causal relationships.

We conclude that cardiac dysfunction, the need for dialysis, and pneumonia are determinants for weaning failure in patients undergoing long-term mechanical ventilation after cardiac surgery. 


\section{ACKNOWLEDGEMENTS}

We wish to thank Julia Fukushima for her help with the statistical analysis and Laura Blackmore for her revision of the manuscript.

\section{REFERENCES}

1. Butler R, Keenan SP, Inman KJ, Sibbald WJ, Block G. Is there a preferred technique for weaning the difficultto-wean patient? A systematic review of the literature. Crit Care Med 1999; 27: 2331-6. (Review)

2. Rady MY, Ryan T. Perioperative predictors of extubation failure and the effect on clinical outcome after cardiac surgery. Crit Care Med 1999; 27: 340-7.

3. Sulzer CF, Chiolero R, Chassot PG, Mueller XM, Revelly JP. Adaptive support ventilation for fast tracheal extubation after cardiac surgery: a randomized controlled study. Anesthesiology 2001; 95: 1339-45.

4. Elpern EH, Larson R, Douglass P, Rosen RL, Bone RC. Long-term outcomes for elderly survivors of prolonged ventilator assistance. Chest 1989; 96: 1120-4.

5. Wong DT, Cheng DC, Kustra R, et al. Risk factors of delayed extubation, prolonged length of stay in the intensive care unit, and mortality in patients undergoing coronary artery bypass grafts with fast-track cardiac anesthesia: a new cardiac risk score. Anesthesiology 1999; 91: 936-44.

6. LoCicero J 3rd, McCann B, Massad M, Joob AW. Prolonged ventilatory support after open-heart surgery. Crit Care Med 1992; 20: 990-2.

7. Nava S, Rubini F, Zanotti E, et al. Survival and prediction of successful ventilator weaning in COPD patients requiring mechanical ventilation for more than 21 days. Eur Respir J 1994; 7 : 1645-52.

8. Gluck EH. Predicting eventual success or failure to wean in patients receiving long-term mechanical ventilation. Chest 1996; 110: 1018-24.

9. Zanotti E, Rubini F, Iotti G, et al. Elevated static compliance of the total respiratory system: early predictor of weaning unsuccess in severed COPD patients mechanically ventilated. Intensive Care Med 1995; 21: 399-405.

10. Nozawa E, Kobayashi E, Matsumoto ME, Feltrim MI, Carmona MJ, Auler Jr. Assessment of factors that influence weaning from long-term mechanical ventilation after cardiac surgery. Arq Bras Cardiol 2003; 80: 301-10.

11. Parsonnet V, Dean D, Bernstein AD. A method of uniform stratification of risk for evaluating the results of surgery in acquired adult heart disease. Circulation 1989; 79: (suppl I): 3-12.

12. Knaus WA, Draper EA, Wagner DP, Zimmerman JE. APACHE II: A severity of disease classification system. Crit Care Med 1985; 13: 818-29.

13. Kollef MH, Wragge T, Pasque C. Determinants of mortality and multiorgan dysfunction in cardiac surgery patients requiring prolonged mechanical ventilation. Chest 1995; 107: 1395-401.

14. Doering LV. Relationship of age, sex, and procedure type to extubation outcome after heart surgery. Heart Lung 1997; 26: 439-47.

15. Wahl GW, Swinburne AJ, Fedullo AJ, Lee DK, Bixby K. Long-term outcome when major complications follow coronary artery bypass graft surgery. Recovery after complicated coronary artery bypass graft surgery. Chest 1996; 110: 1394-8.

16. Engoren M, Buderer NF, Zacharias A. Long-term survival and health status after prolonged mechanical ventilation after cardiac surgery. Crit Care Med 2000; 28: 2742-9.

17. Ferraris VA, Ferraris SP. Risks factors for postoperative morbidity. J Thorac Cardiovasc Surg 1996; 111: 73138; discussion 738-41.

18. Weintraub WS, Jones EL, Craver J, Guyton R, Cohen C. Determinants of prolonged length of hospital stay after coronary bypass surgery. Circulation 1989; 80: 276-84.

19. O'Connor GT, Plume KS, Olmstead EM, et al. Multivariate prediction of in-hospital mortality associated with coronary artery bypass graft surgery. Northern New England Cardiovascular Disease Study Group. Circulation 1992; 85: 2110-8.

20. van Belle AF, Wesseling GJ, Penn OC, Wouters EF. Postoperative pulmonary function abnormalities after coronary artery bypass surgery. Respir Med 1992; 86: 195-9. 
21. Ratliff NB, Young WG Jr, Hackel DB, Mikat E, Wilson JW. Pulmonary injury secondary to extracorporeal circulation. An ultrastructural study. J Thorac Cardiovasc Surg 1973; 65: 425-32.

22. Auler JO Jr, Carmona MJ, Barbas CV, Saldiva PH, Malbouisson LM. The effects of positive end-expiratory pressure on respiratory system mechanics and hemodynamics in postoperative cardiac surgery patients. Braz $\mathrm{J}$ Med Biol Res 2000; 33: 31-42.

23. Hess DH. Mechanical ventilation strategies: what's new and what's worth keeping? Respir Care 2002; 47: 1007-17. (Review)

24. Kollef MH, Sharpless L, Vlasnik J, Pasque C, Murphy D, Fraser VJ. The impact of nosocomial infections on patient outcomes following cardiac surgery. Chest 1997; 112: 666-75.

25. Bouza E, Perez A, Munoz P, et al. Ventilator-associated pneumonia after heart surgery: a prospective analysis and the value of surveillance. Crit Care Med 2003; 31: 1964-70.

26. Landymore RW, Howell F. Pulmonary complications following myocardial revascularization with the internal mammary artery graft. Eur J Cardiothorac Surg 1990; 4: 156-61; discussion 161-2.

27. Magnusson L, Zemgulis V, Wicky S, Tyden H, Thelin S, Hedenstierna G. Atelectasis is a major cause of hypoxemia and shunt after cardiopulmonary bypass: an experimental study. Anesthesiology 1997; 87: 1153-63.

28. Singh NP, Vargas FS, Cukier A, Terra-Filho M, Teixeira LR, Light RW. Arterial blood gases after coronary artery bypass surgery. Chest 1992; 102: 1337-41.

29. Tenling A, Hachenberg T, Tyden H, Wegenius G, Hedenstierna G. Atelectasis and gas exchange after cardiac surgery. Anesthesiology 1998; 89: 371-8.

30. Isgro F, Schmidt C, Pohl P, Saggau W. A predictive parameter in patients with brain related complications after cardiac surgery? Eur J Cardiothorac Surg 1997; 11: 640-4.

31. Roach GW, Kanchuger M, Mangano CM, et al. Adverse cerebral outcomes after coronary bypass surgery. Multicenter Study of Perioperative 1schemia Research Group and the Ischemia Research and Education Foundation Investigators. N Engl J Med 1996; 335: 1857-63.

32. Bojar RM, Najafi H, DeLaria GA, Serry C, Goldin MD. Neurological complications of coronary revascularization. Ann Thorac Surg 1983; 36: 427-32.

33. Abel RM, Buckley MJ, Austen WG, et al. Etiology, incidence, and prognosis of renal failure following cardiac operations. Results of a prospective analysis of 500 consecutive patients. J Thorac Cardiovasc Surg 1976; 71: 323-3.

34. Chertow GM, Levy EM, Hammermeister KE, Grover F, Daley J. Independent association between acute renal failure and mortality following cardiac surgery. Am J Med 1998; 104: 343-8. 